Bull. Mater. Sci., Vol. 17, No. 6, November 1994, pp. 595-606. (O Printed in India.

\title{
Flux trapping and its effect on AC susceptibility of granular superconductors
}

\author{
SUBIR SAHA and B K DAS \\ Materials Division, National Physical Laboratory, Dr K S Krishnan Road, New Delhi 110012 , \\ India

\begin{abstract}
Experimental data on the real $\left(\chi^{\prime}\right)$ and imaginary $\left(\chi^{\prime}\right)$ parts of AC magnetic susceptibility as a function of DC bias field $(H)$ shows the effect of trapped flux in granular high $T_{\mathrm{c}}$ superconductors. The aim was to substantiate our recent theoretical findings on the basis of a two-component critical state model suitable for granular high $T_{\varepsilon}$ superconductors. Stress has been given to understanding the origin of hysteresis in $\chi^{\prime}(H)$ and $\chi^{\prime \prime}(H)$. It was seen in the experimental data that above a certain value of DC field range irreversibility appears in $\chi^{\prime}(H)$ and $\chi^{\prime \prime}(H)$ creating hysteresis like loops. Comparison of data with calculated loops shows good agreement. $\chi^{\prime}(H)$ and $\chi^{\circ}(H)$ curves show considerable asymmetry in presence of trapped flux.
\end{abstract}

Keywords. Susceptibility; superconductors; critical state.

\section{Introduction}

Understanding the nature of the flux line lattice (FLL) and the flux dynamics in the superconducting ceramics, i.e. granular superconductors, has posed a serious challenge to the theoreticians as well as experimental workers. A lot of experimental work has been done in this regard to substantiate our understanding but an agreeable understanding is far from our reach. The so called phase diagram of the vortices which has been accepted for conventional superconductors with confidence has been looked upon with confusion (Fisher et al 1990). The presence of an irreversibility line has led to complicated situation. Explanation of this irreversibility line is not yet well accepted. From microscopic point of view, the inclusion of small coherence length, higher critical temperature and anisotropy will have to be considered in a single model. However, such a model is yet to come. Most models are based on Bean's critical state model (Bean 1962, 1964) for flux penetration into a superconductor. Such models (Clem 1988; Bhagawat and Chaddah 1989; Tinkham and Lobb 1989; Müller 1990; Saha and Das 1993a; Chaddah 1994) have been partially successful in explaining the magnetic properties of granular high $T_{\mathrm{c}}$ superconductors.

AC susceptibility measurement has been often used just to characterize the critical temperature and the superconducting volume fraction. However a careful measurement of the in-phase and out-of-phase components $\left(\chi^{\prime}\right.$ and $\left.\chi^{\prime \prime}\right)$ of AC susceptibility provides more insight into the nature of flux dynamics. Measurement of $\mathrm{AC}$ susceptibility as a function of temperature for different $\mathrm{AC}$ amplitudes and superimposed DC fields has provided valuable information on the granularity of the high $T_{c}$ superconductors (Küpfer et al 1988).

Measurements of AC susceptibility as a function of DC bias field is also important and can lead to better understanding of the problem. Though there are some results 
on field dependence of $\chi^{\prime}$ and $\chi^{\prime \prime}$ and hysteresis thereof (Strongin and Maxwell 1963; Goldfarb and Clark 1985; Nieuwenhuys et al 1988; Kompany et al 1990; Ciszek et al 1991; Lee et al 1992), a thorough study in this line is still important. Moreover in most of the AC susceptibility studies the DC and AC fields are parallel which allows one to exploit the critical state model in explaining the data (Campbell et al 1983) because in that case one can consider the fields as scaler quantities and hence a simple one-dimensional critical state model is sufficient for the purpose. A more involved model has been recently suggested by Bhagawat et al (1994). In presence of a DC bias field, one has to consider two concurrent processes-firstly, the effect of DC biasing in modifying the effective $J_{c}$, and so the variation of $\chi^{\prime}$ and $\chi^{\prime \prime}$ as a function of DC bias and secondly, the flux reorganization within a given $\mathrm{AC}$ cycle and consequent losses. The effective situation is-major $M-H$ loops due to DC biasing and superposition of minor $M-H$ loops due to $\mathrm{AC}$ field at different $\mathrm{DC}$ bias fields. In order to get a good simulation of experimental data any model calculation must handle this carefully. One must consider the experimental situation in detail, i.e. how the AC and DC fields are being imposed. There may be two situations, viz. (i) the AC field remains on while the DC field is being ramped and (ii) the AC field is put on only after coming to a certain value of the DC field where measurements are to be done. It may appear that this does not make much difference. However, a close. look will bring out the difference in the two cases. If the $\mathrm{AC}$ amplitude is higher than the step size of the DC field variation then in both cases the resultant flux profile (i.e. the DC profile modified by the $\mathrm{AC}$ oscillation) and hence the resultant major loop will be nearly the same. But if the step is larger than the AC amplitudes then in the second case the major loop will be disturbed by $\mathrm{AC}$ fields at the point of measurement only and hence the two cases will be different. As one can see Saha and Das (1993b) model calculation resembles the proposition of the 2nd type and hence at lower amplitude additional hysteresis can be seen even without further correction and which usually are not seen in the data of conventional superconductors (Goldfarb and Clark 1985).

\section{Experimental details}

AC magnetic susceptibility of samples were measured by the mutual induction method. The two secondaries were connected in series opposition and coaxial to the primary which was coiled on a separate former. The two secondaries were separated by $2 \mathrm{~mm}$ and each had 450 turns while the primary consisted of 500 turns (wire diameter $0.08 \mathrm{~mm}$ ). The total secondary length was only $10 \mathrm{~mm}$ while the primary was of $40 \mathrm{~mm}$ length. The DC bias field was generated by a third coaxial solenoid (15 cm in length) and had 10 layers of $39 \mathrm{BSW}$ wire. Maximum field that could be generated was $20 \mathrm{mT}$ and was driven by an analog voltage ramp as well as a programmable DC source. The whole assembly could be dipped in a liquid nitrogen bath. Samples were cut into cylinders of nominal diameter $\approx 1-2 \mathrm{~mm}$ and length $(\approx 2-3) \mathrm{mm}$. The sample was put into a quartz bucket held in one of the secondaries and the resultant signal was picked up by a two-phase lock-in-amplifier. This signal was monitored against the field current through the DC bias coil. It must be noted that the experimental situation was that of first 
case, i.e. AC field was continuously on. In absence of a balancing circuit, it was not possible to have exact balance at a given frequency. However, by exploiting the non-uniformity in the primary field one could achieve quite a bit of balancing by moving the secondary pairs within the primary. The rest of the signal was offsetted by using the built-in facility of LIA or through software. In order to bring out possible significance from these arbitrary measurements all the data were offsetted at the highest DC bias field and then plotted.

\section{Model calculations}

The method of calculation is described elsewhere and will not be discussed here (Saha and Das 1993b). To bring out better fit to the data, the step size of DC bias field is kept quite less $(0.01 \mathrm{mT})$ during model calculations to have a closer resemblance with experimental situation and hence the calculation as presented here should not be confused with that of Saha and Das (1993b) where the step size was 0.1 or $2.0 \mathrm{mT}$. Further, as we shall consider the low field data only, we will not consider demagnetization contribution due to grains. In order to understand the basic nature of data we consider a higher bulk demagnetization factor of 0.9 instead of 0.3 as considered in Saha and Das (1993b).

\section{Results and discussion}

\subsection{Range effect of DC bias field}

In figures $1 \mathrm{a}$ and $\mathrm{b}$ the real $\left(\chi^{\prime}\right)$ and imaginary $\left(\chi^{\prime \prime}\right)$ parts of $\mathrm{AC}$ susceptibility as a function of DC bias field have been plotted for a pure YBCO sample. The amplitude of $\mathrm{AC}$ excitation was $0.3 \mathrm{mT}$, the highest amplitude employed in this work. In interpreting these results it has to be remembered that the measurements were in arbitrary units and that the individual curves were arbitrarily zero offsetted at the highest DC bias field of excursions. Because of the relative measurements, it was not always possible to compare the graphs for different parameters directly. However, in a given frame (diagram) all the curves were plotted in the same scale and can be compared for the change of shapes for different parameters, i.e. range of DC bias field, amplitude of AC excitation etc.

The important findings in figures $1 \mathrm{a}$ and $\mathrm{b}$ are that (i) below a maximum of the DC field excursion there is no hysteresis seen either in real or imaginary parts and (ii) with the increase of the range of DC field excursion, the hysteresis in real parts increases monotonically, whereas in imaginary parts new features are seen to appear along with increase of hysteresis. The striking feature, that is seen in all the cases at the zero cross over points-the sharp change in $\chi^{\prime}$ and $\chi^{\prime \prime}$ near $H=0-$ is being reported for the first time. It is difficult to explain the origin of this feature. Moreover this zero cross over feature (ZCOF) is not seen in the model calculations (Saha and Das 1993b). In the first instant, one obviously will consider $H_{\text {clj }}$ of the grain boundaries responsible for ZCOF. However, a close look rules out this possibility. Because, by arguments of Campbell et al (1983), the feature at $H_{\mathrm{c} 1}$ will be negligible when $\mathrm{DC}$ ramp, i.e. 

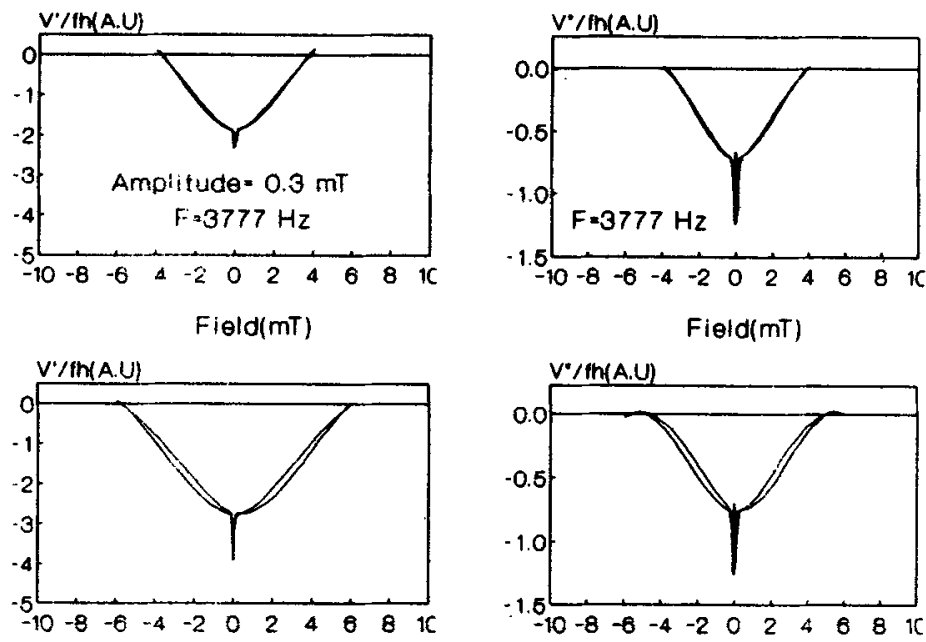

Field(mT)

Field(mT)
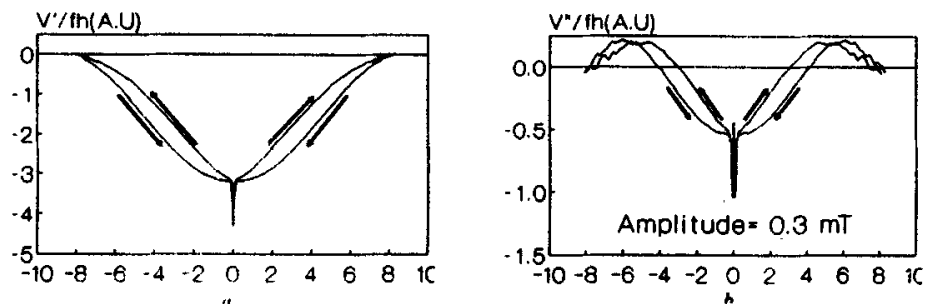

Figure 1. a. $I^{\prime} / h\left(\approx \chi^{\prime}\right)$ as at lunction of $D C$ bits ficld for three different ranges of $D C$ ficld and b. $V^{\prime \prime} / f h\left(\approx \chi^{\prime \prime}\right)$ as a function of $D C$ bias lield for three different ranges of DC ficld.

$$
\mathrm{d} H_{\mathrm{dc}} / \mathrm{d} t \ll H_{\mathrm{uc}} \omega
$$

In our experiments, the ramp used becomes slower at cross over region and above condition is further true in this region. It is seen by zooming the cross over region that within experimental error, the ZCOF is a symmetric peak centred at $H_{\mathrm{dc}}=0$ instead of a non-zero DC field. In a later communication, it will further be shown that ZCOF becomes more prominent with increasing frequency which is also in contradiction to Campbell et al (1983).

The presence of ZCOF as reported here and not seen in the already published data of others (Nieuwenhuys 1988; Kompany et al 1990; Ciszek et al 1991; Yamaguchi et al 1991: Lee et al 1992) can be understood due to the fact that in the experimental set-up one usually employs a quasistatic variation of DC field using a constant current source (programmable), whereas here a continuous voltage ramp was utilized to ramp the DC field. A constant current source, which drives the inductive load of the magnet in a feedback loop, cannot provide a true zero current and hence in this kind of arrangement the ZCOF being sharp and close to zero bias field, will be missed. It was in fact verified in the experiments that the ZCOF is not present when the DC field variation is quasistatic. However, at lower 

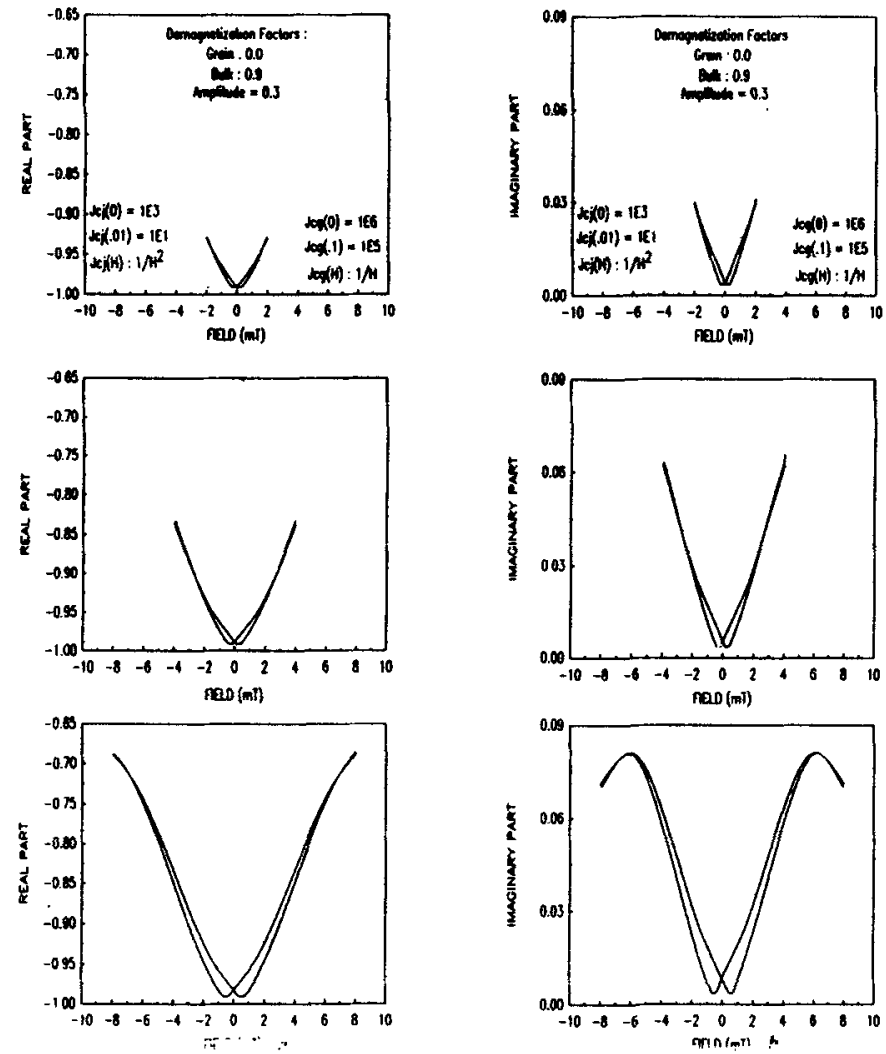

Figure 2. a. Calculated $\chi^{\prime}(H)$ for three DC bias ranges with intragrain $\left(J_{\mathrm{cg}}\right)$ and intergrain $\left(J_{\mathrm{cj}}\right)$ parameters (in $\mathrm{A} / \mathrm{cm}^{2}$ ) and their field dependence as shown and b. calculated $\chi^{\prime \prime}(H)$ for three different DC bias ranges with parameters of a.

frequencies (e.g. $373 \mathrm{~Hz}$ ) even in the quasistatic case a small ZCOF is seen (it is the result of the above mentioned frequency dependence of ZCOF). Though, ZCOF is an interesting feature in our data, in our further discussion in this work we shall avoid this complexity.

The other important feature of the real loops is that there is no change in shape of the curves, but the depths (i.e. the depth of the valley excluding ZCOF) of these loops (figure 1) increase with increase in the range of DC field. However, in case of $\chi^{\prime \prime}(H)$ loops, there is a substantial change in the shape of the curves with a peak seen to appear (bottom most loop in figure $1 b$ ) at high $\mathrm{DC}$ bias field values for the largest range of DC bias field. For $\chi^{\prime \prime}(H)$ loops as can be seen in figure $1 \mathrm{~b}$, the heights (the term 'height' is introduced in view of the fact that if the loops are not offsetted they would be in +ve side of the $y$-axis) of these curves, excluding ZCOF, i.e. the separation between the minimum (excluding ZCOF) and the maximum of the curves are more or less independent of the variation of the range of DC field. However, the described behaviour of $\chi^{\prime}$ and $\chi^{\prime \prime}$ loops is very much sample dependent and is not same in different samples.

The explanation of this dependence on the range of DC field can be given on 

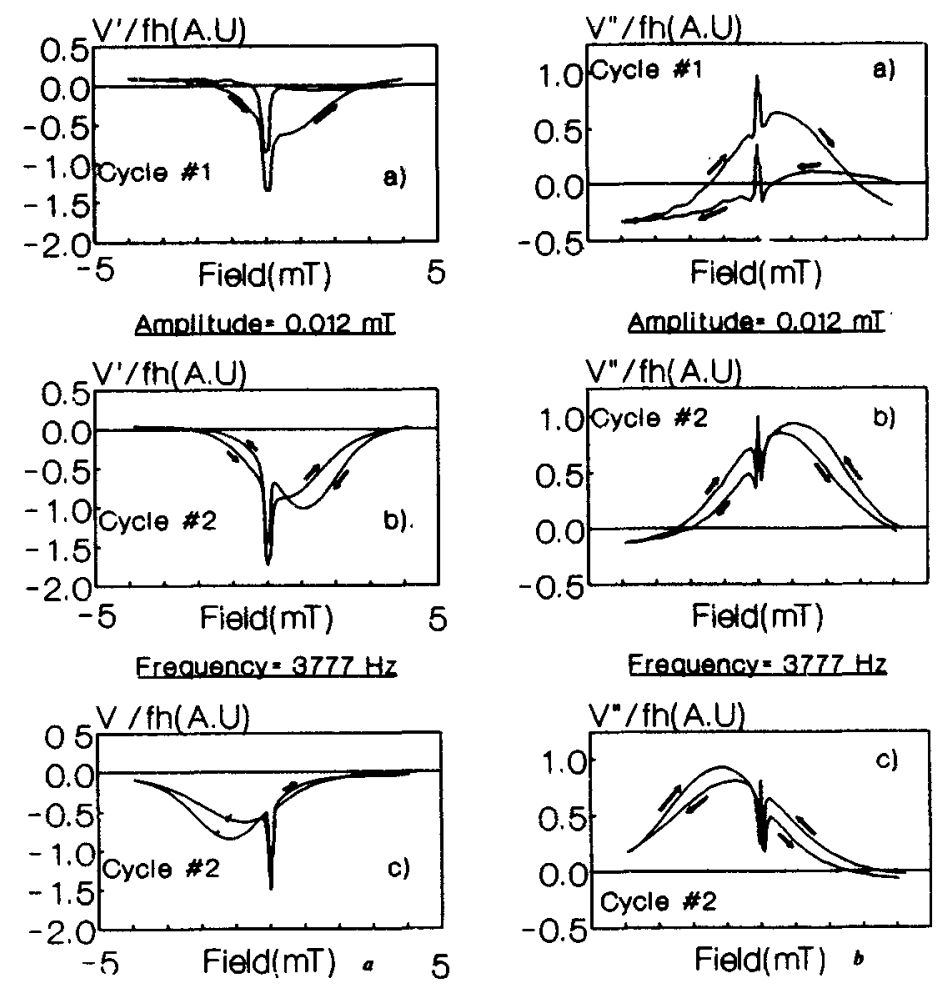

ligure 3. a. $V^{\prime} / h$ as a function of $I X$ bias field after applying a trapping licld and $b$. $V " / f h$ as a function of DC bias field after applying a trapping field.

the basis of the model calculations as given by Saha and Das (1993b). Such a calculation has been reproduced in figure 2 with intergrain and intragrain critical current density, arameters as indicated. Though a qualitative agreement is apparent any disagreement between the model calculations and the experimental data is obviously due to the fact that the model still is an over simplified representation of the actual physical situation. It must be noted that the calculation has correctly brought out the essential features of the data in figure 1, e.g. (i) the increase in hysteresis with increasing DC field range and (ii) appearance of peaks on the $\chi^{\prime \prime}(H)$ curves. As one can understand from figure 2, with increasing DC field a larger amount of flux gets trapped giving rise to higher and higher hysteresis. In the model, this has been tackled by considering a field dependent demagnetization factor contribution. That, this hysteresis is due to the trapping of flux lines could further be ascertained from the following experiments. After taking the DC field to a maximum of $+20 \mathrm{mT}$, the same $\chi^{\prime}(H)$ and $\chi^{\prime \prime}(H)$ loops are drawn for lower field ranges $( \pm 5 \mathrm{mT})$ as shown in figure $3(\mathrm{a}-\mathrm{b})$ for amplitude of $0.012 \mathrm{mT}$ for a $\mathrm{Pb}$ doped YBCO sample with $J_{\mathrm{cj}}$ (transport $J_{\mathrm{c}}$ ) lower than that of pure YBCO. As can be seen in these diagrams, the loops in the cycle \#1 $(+20 \mathrm{mT}$ to $5 \mathrm{mT}$ and then $+5 \mathrm{mT}$ ) are quite different, because in this cycle there is large flux reorganization. However, in cycle \#2 $( \pm 5 \mathrm{mT})$, only some excess flux remains 

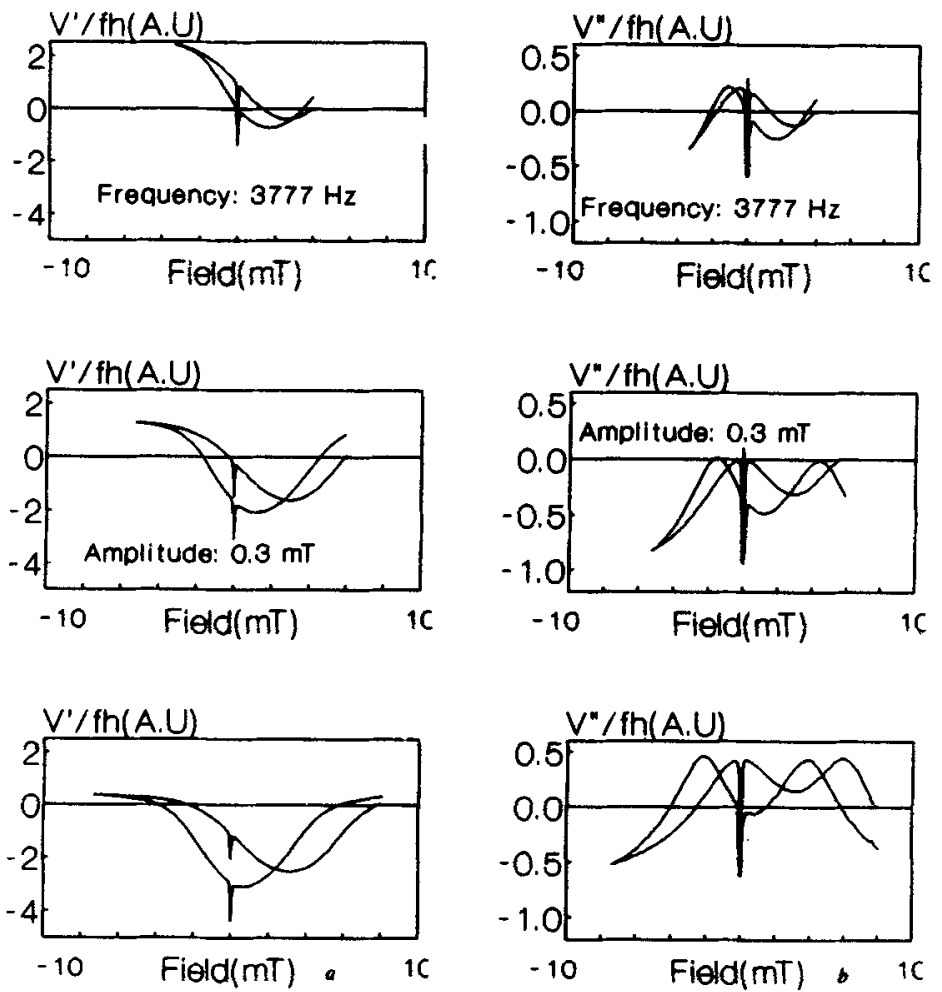

Figure 4. a. $V^{\prime} / / h$ as a function of $D C$ bias field for different ranges of $D C$ bias (cycle $\# 1)$ and b. $V^{\prime \prime} / \mathrm{fh}$ as a function of DC bias field for different ranges of DC bias (cycle $\# 1$ ).

trapped which distorts the symmetry of the loops (curve $b$ of figures $3 a$ and $b$ ). That this asymmetry is certainly due to the trapped flux could be ascertained from a reverse field excursion (i.e. starting from $-20 \mathrm{mT}$ and then cycling $\pm 5 \mathrm{mT}$ ) as seen in curves $c$ of figures $3 a$ and $b$ where the asymmetry is reversed. It is further important to consider the effect of range on this trapped flux. In figures $4 a$ and $\mathrm{b}$ (cycle \#1), and figures $5 \mathrm{a}$ and $\mathrm{b}$ (cycle \#2) the $\chi^{\prime}(H)$ and $\chi^{\prime \prime}(H)$ loops have been plotted for three different field ranges $( \pm 4, \pm 6$, and $\pm 8 \mathrm{mT}$ in cycles \#1 and \#2) starting from a higher DC field of $+20 \mathrm{mT}$. Here the amplitude was $0.3 \mathrm{mT}$ and sample was having $J_{\mathrm{cj}}$ in between the previous two samples. In comparison with figure 3, it can be seen here that with higher amplitude, the disturbance as well as hysteresis in cycle \#l is less at all ranges. This is plausible, because with higher amplitude, flux would be averaged over a larger volume during the AC cycling leaving less flux reorganization to occur over the DC field cycling. However, even at this higher amplitude, the disturbance in cycle \#1 in figure 4 at all ranges is more than that in cycle \#2 in figure 5. It is clear from these figures that though the asymmetry is present in all the cases, it is less with higher ranges-a physically obvious expectation. Moreover, this additional trapped flux acts like a bias field only and does not contribute to hysteresis as can be seen in the cycle \#2 data where at the lowest field range no hysteresis is seen. 

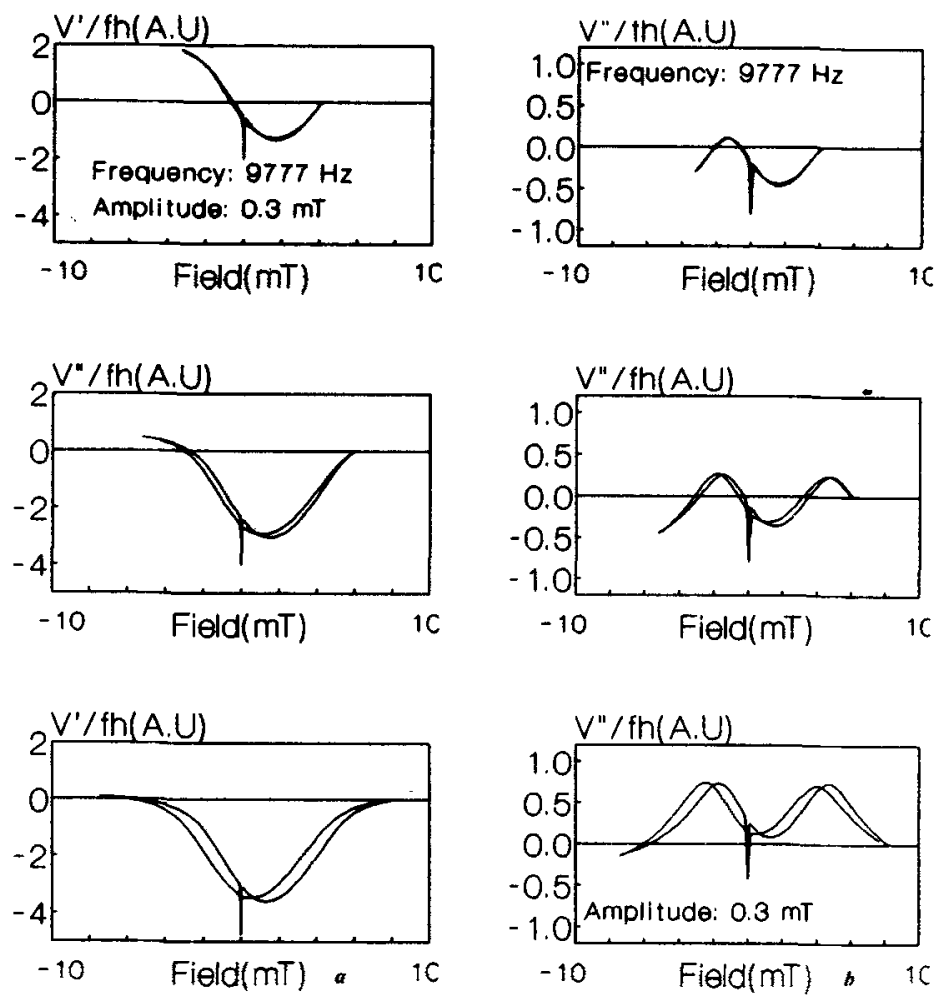

Figure 5. a. $V^{\prime} / / h$ as a function of $1 \mathrm{CC}$ bias field for different ranges of DC bias (cycle $\# 2$ ) and b. $V^{\prime \prime} / f h$ as a function of $D C$ bias ficld for different ranges of DC bias (cycle \#2).

\subsection{Amplitude dependence of the $\chi^{\prime}(H)$ and $\chi^{\prime \prime}(H)$ loops}

The dependence of $\chi^{\prime}$ and $\chi^{\prime \prime}$ loops on amplitude of the AC excitation field is quite large and provides rich information vide comparison with the model calculations. However, only three amplitudes have been considered here and they seem to cover a wide range of behaviour of the loops and show a systematic variation.

Figures 6a and b give $\chi^{\prime}(H)$ and $\chi^{\prime \prime}(H)$ for a DC field range of $\pm 18 \mathrm{mT}$. In the real parts, as can be seen in figure $6 a$ there is a decrease in field dependence with increase in AC amplitude and otherwise the loops are quite monotonic. But the $\chi^{\prime \prime}(H)$ loops show quite striking changes. The general feature of this $\chi^{\prime \prime}(H)$ loops is that there is a valley $(V)$ on the decreasing arms in both sides and two peaks $(P 1$ and $P 2)$ on each arm at a finite DC bias (indicated in figure $6 \mathrm{~b}$ ). The position of the peaks ( $P 1$ and $P 2)$ are seen to be very much dependent on amplitude and moves to higher DC bias (magnitude wise) side with decreasing amplitude. Even, if the amplitude is quite high, the peaks, $P$ s coincide and as a consequence the valleys, Vs cannot be seen. This movement of the peaks with amplitude variation causes an apparent shift of the valley upwards as seen in figure $6 \mathrm{~b}$.

In an effort to simulate this data from our model calculation, figures $7 a$ and $b$ are reproduced here with parameters as shown. As one can see, the agreement 

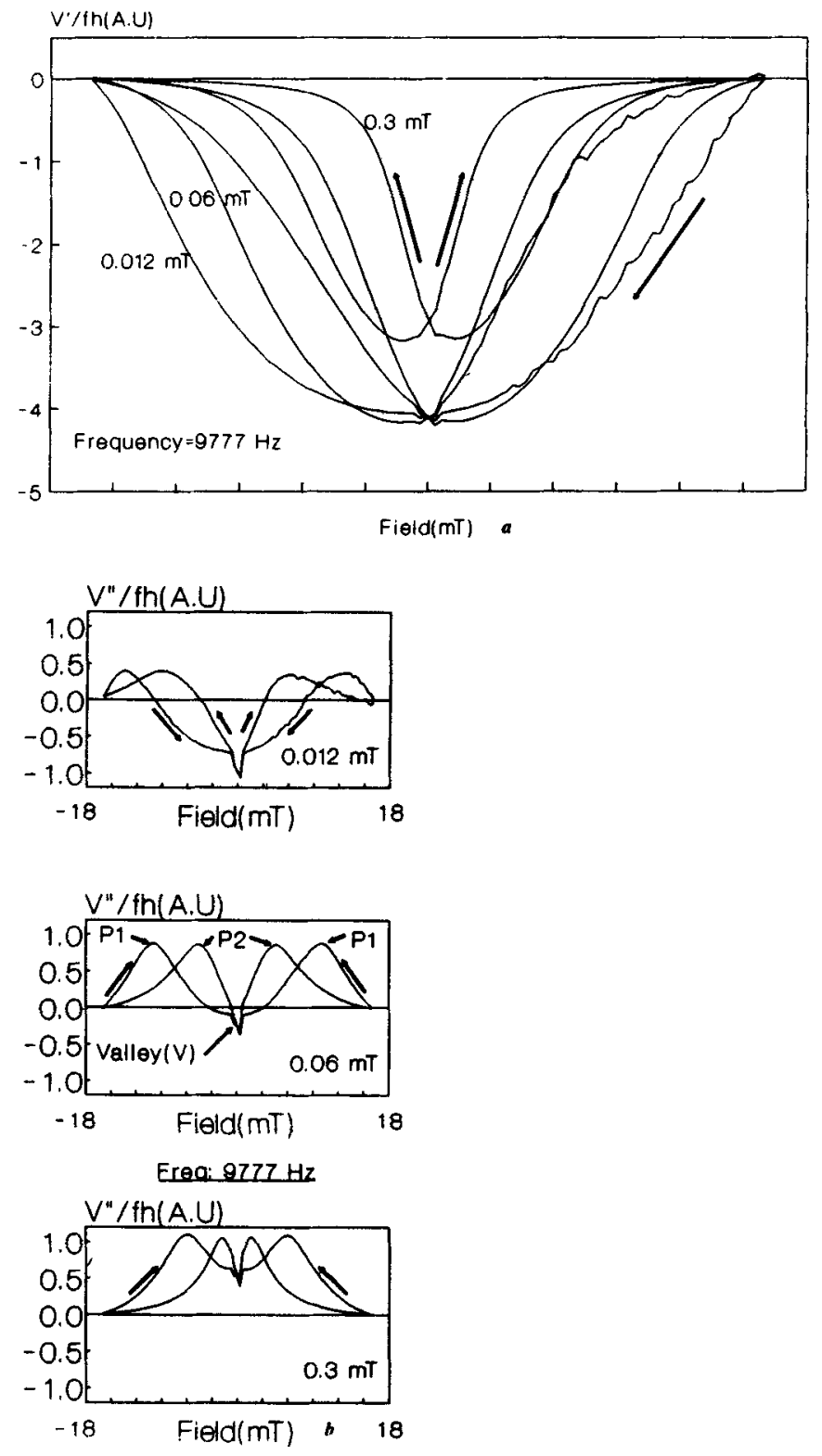

Figure 6. a. $V / j h$ as a function of DC bias field for different AC amplitudes (DC bias field range: $\pm 18 \mathrm{mT}$ ) and $b . V " / f h$ as a function of $\mathrm{DC}$ bias field for different amplitudes.

between results and data is quite striking. In figure $7 \mathrm{a}$, the $\chi^{\prime}(H)$ loops resemble that of figure $6 \mathrm{a}$ and show the same tendency, i.e. valleys move upward with increasing amplitude. In $\chi^{\prime \prime}(H)$ loops (figure $7 \mathrm{~b}$ ), the movement of peaks towards higher values of DC field with decreasing amplitude shows a behaviour similar to the data in figure $6 \mathrm{~b}$. At the same time, the valleys, in the calculated $\chi^{\prime \prime}(H)$ loops are also seen to move upward with increasing amplitudes. In fact, if one can 

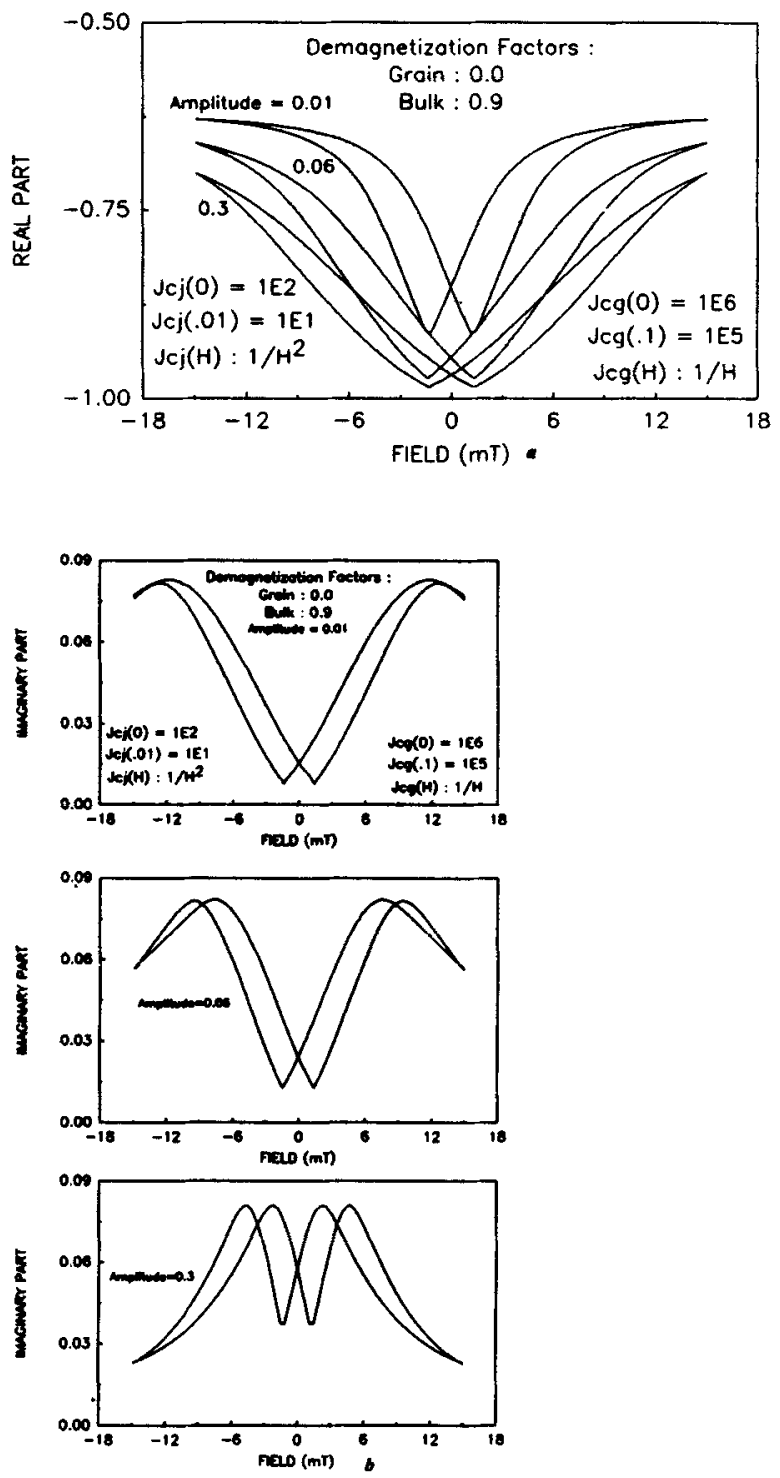

Figure 7. a. Calculated $\chi^{*}(H)$ for different $A C$ amplitudes with $J_{c}$ parameters as shown taking $J_{\mathrm{cj}} \sim 1 / H^{2}$ and $J_{\mathrm{cg}} \sim 1 / H$ and b. calculated $\chi^{\prime \prime}(H)$ for different AC amplitudes with parameters of $\mathbf{a}$.

produce absolute measurements in these experiments, it may be possible to compare the sample properties in a rigorous way.

Another aspect of the $\chi^{\prime \prime}(H)$ loops for different amplitudes as seen here is that with lower amplitude the split of the increasing and decreasing arms of the loop, $\Delta H$, on each side (+ve or -ve $H$ sides) is larger. This experimental finding is also justified in view of our results (figure $7 \mathrm{~b}$ ). 

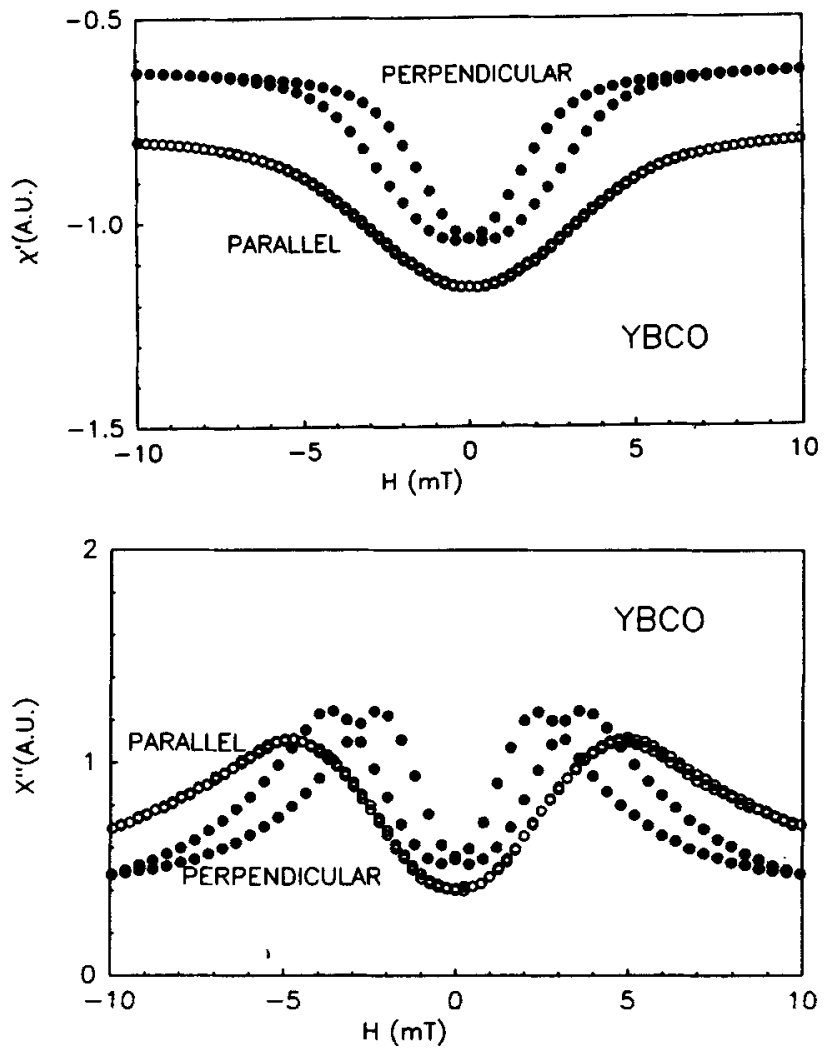

Figure 8. Measured $\chi^{\prime}(H)$ and $\chi^{\prime \prime}(H)$ of a YBCO sample when AC and DC fields are parallel or perpendicular. Note the increase in hysteresis in perpendicular case.

However, the obvious disagreement with the model calculations is that the calculations could not reproduce the amount of hysteresis in $\chi^{\prime \prime}(H)$ loops observed experimentally. The obvious limitation of the model is that it is a one-dimensional one and cannot account for the curved flux lines inside the superconductor, which in actual situation of granular system cannot be avoided. Further, the current crowding in these materials must also be considered in a more realistic way than the simple approach as considered in the model of Saha and Das (1993b) by way of field dependent demagnetization.

\subsection{Perpendicular condition}

When the $\mathrm{AC}$ and $\mathrm{DC}$ fields are perpendicular, the flux lines cannot be along the axis of the sample and flux line lattice has to be treated by the method recently given by Bhagawat et al (1994). Experimental measurements done by Dhingra and Das (1994) on a YBCO sample with AC amplitude of $0.27 \mathrm{mT}$ shows that the hysteresis in $\chi^{\prime}(H)$ and $\chi^{\prime \prime}(H)$ increases as one goes from the parallel configuration to the perpendicular configuration as shown in figure 8. Besides the complexity of flux line bending/rotation during AC excitation, we have to consider the fact that the geometry of the sample was such that the demagnetization factor of the sample was higher in the perpendicular configuration. 


\section{Conclusions}

Dependence of AC magnetic susceptibility on the superimposed DC magnetic field for granular high $T_{c}$ superconductors shows considerable hysteresis even at low DC bias fields. The shape of the curves depends on the inter- and intra-grain critical current densities, the demagnetization factors of the grains and the sample and the orientation of the AC and DC fields. Trapped flux, if any, also influences the shape of the $\chi^{\prime}(H)$ and $\chi^{\prime \prime}(H)$ curves. Therefore, extreme care has to be taken while interpreting the susceptibility data to arrive at $J_{c} s$. One-dimensional two-component critical state model can explain general features of the $\chi^{\prime}(H)$ and $\chi^{\prime \prime}(H)$ curves, but a quantitative match between the experimental data and theoretical predictions can only be possible when more intricate models evolve.

\section{Acknowledgements}

One of the authors (SS) thanks CSIR for providing financial assistance. Authors acknowledge help from Drs P Chaddah and A K Grover in the form of stimulating discussions and Dr I Dhingra for providing some of her unpublished results.

\section{References}

Bean C P 1962 Phys. Rev. 38250

Bean C P 1964 Rev. Mod. Phys. 3631

Bhagawat K V and Chaddah P 1989 Pramana-J. Phys. 33521

Bhagawat K V, Nair S V and Chaddah P 1994 Physica C227 176

Campbell S A, Ketterson J B and Crabtree G W 1983 Rev. Sci. Instrum. 541191

Chaddah P in Studies of high temperature superconductors (ed.) A V Narlikar (New York: Nova Science Pub.) 14

Ciszek M, Zaleski A J, Olejniczak J and van de Klundert L J M 1991 Physica C185-189 2135

Clem J R 1988 Physica C153-155 50

Dhingra I and Das B K 1994 (Unpublished data)

Fisher D S, Fisher M P A and Huse D A 1990 Phys Rev. B43 130

Goldfarb R B and Clark A F 1985 IEEE Trans. MAG 21332

Kompany A, Qian Y J, Xu M F, Baum H P and Levy M 1990 Solid State Commun. 75579

Küpfer $H$ et al 1988 Cryogenics 28650

Lee C Y, Song L W and Kao Y H 1992 Physica C191 429

Müller K H 1990 Physica C168 585

Nieuwenhuys G J, Friedmann T A, Rice J P, Gehring P M, Salamon M B and Ginsberg D M 1988 Solid State Commun. 67253

Saha S and Das B K 1993a Supcond. Sci \& Tech. 6685

Saha S and Das B K 1993b Supcond. Sci \& Tech. 6840

Strongin M and Maxwell E 1963 Phys. Lett. 649

Tinkham M and Lobb C J 1989 Solid State Phys. 4291

Yamaguchi Y, Tokumoto M and Mitsugi K 1991 Physica C179 447 